\title{
Simulation of Pulling the Reinforcing Bar from Concrete Block with Account of Friction and Concrete Damage
}

\author{
Mikhail Beliaev ${ }^{1, *}$, Artem Semenov ${ }^{1}$, Sergey Semenov ${ }^{1}$, and Andrey Benin ${ }^{2}$ \\ ${ }^{1}$ Peter the Great Saint-Petersburg Polytechnic University, Polytechnicheskaya st. 29, St. Petersburg, \\ 195251, Russia \\ ${ }^{2}$ Petersburg State Transport University, Moskovsky pr. 9, St. Petersburg, 190031, Russia
}

\begin{abstract}
A direct finite element modeling of the pulling process of the steel reinforcing bar out from the concrete block with taking into account the cohesive behavior of steel-concrete bond and using elastic-plasticdamage constitutive equations for the concrete is considered. The comparison of obtained results of finite element simulations with experimental data is presented and discussed for the problem of pulling the reinforcing bar from the concrete block.
\end{abstract}

\section{Introduction}

One of the main problems in reinforced concrete elements is the contact between concrete body and steel rebars [1]. It allows them operates as a single monolithic body is a reinforced concrete bond. Reducing coupling leads to excessive crack opening, to the decreasing stiffness and to the reduced carrying capacity of the structure $[2,3]$.

Pulling the rebar out of the concrete block shows the process of destroying reinforcedconcrete bond. It is a complex multistage process, accompanied by the inelastic deformation, the cracking and crushing, the rupture of adhesive links, the presence of tribological phenomena and so on $[4,5]$.

The objective in this paper is to compare the various numerical approaches to modeling of pulling the steel rebar out of the concrete block with experimental results.

\section{Materials and Methods}

\subsection{Experiment}

Pulling the reinforcing bar from the concrete block in accordance with RILEM/CEB/FIB [6] requirements is considered. Loading conditions and geometric parameters are shown in Fig. 1, 2. 


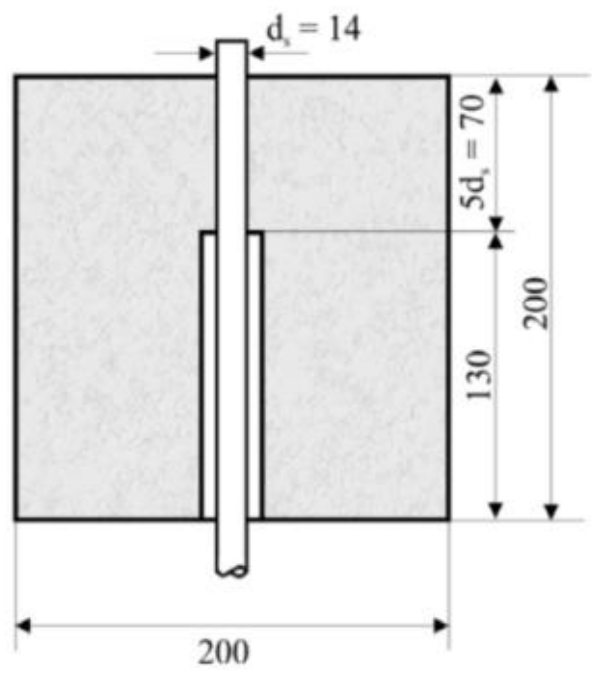

Fig. 1. Geometric parameters of concrete prism and steel rebar;

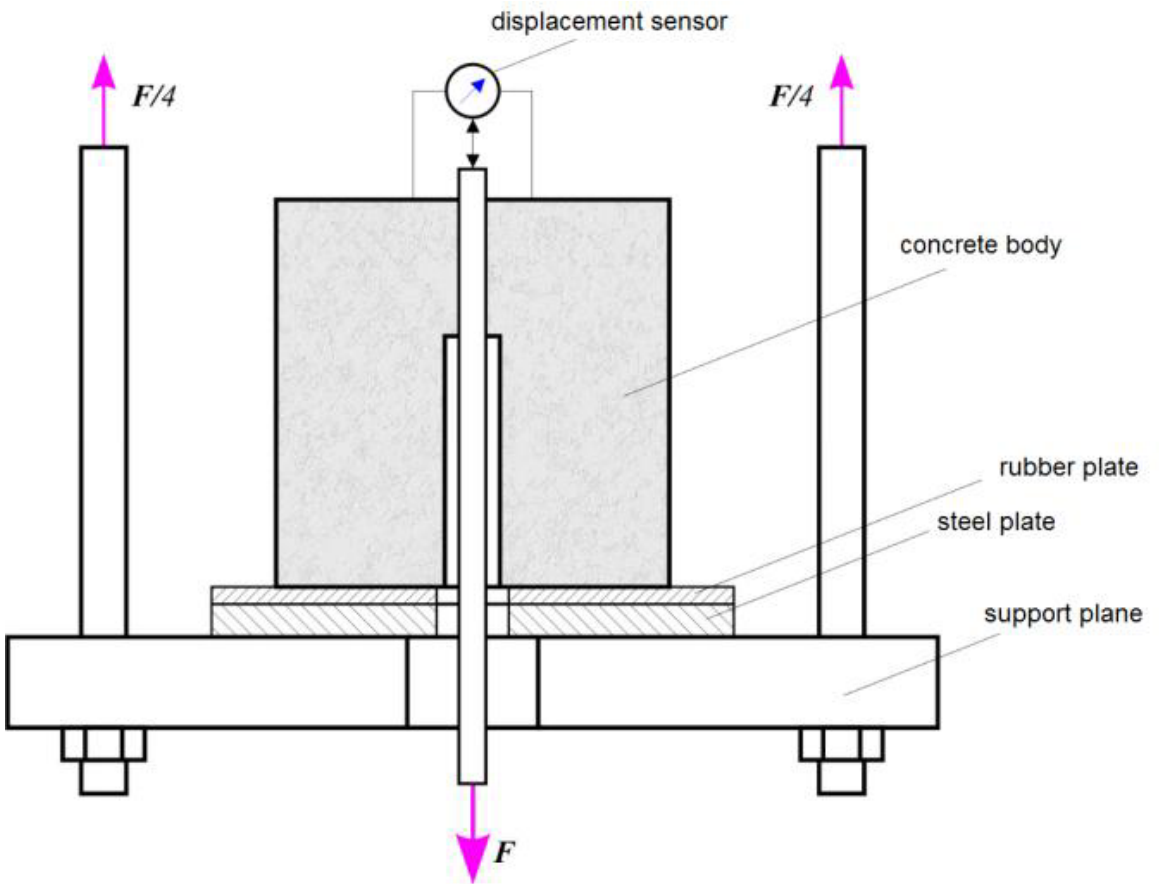

Fig. 2. Loading conditions.

Pull-out test experiment is shown in Fig. 3. It was conducted on tensile tester Shimadzu AG-300kN [7, 8]. Steel reinforcement Ø14 AIII and concrete B25 were used in experiments. 


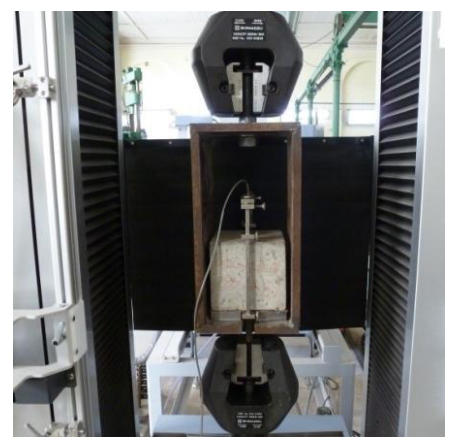

a)

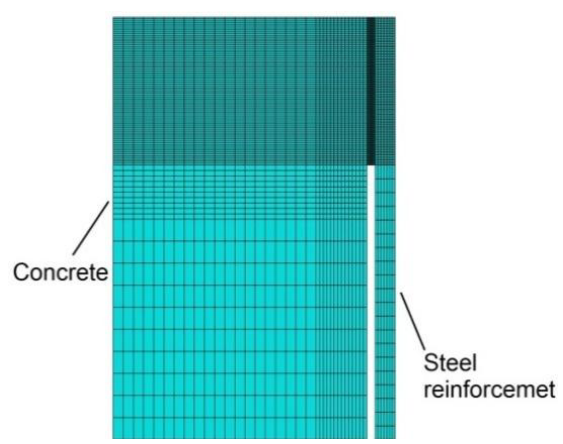

b)

Fig. 3. (a) Tensile tester Shimadzu AG-300kN; (b) 2D FE model.

\subsection{Concrete model}

The elastic, elastic-damage and elastic-plastic damage models are used in the present analysis [5,9-11]. In order to capture the degradation of the elastic stiffness of the concrete material as well as its irreversible deformations upon mechanical loading, the combined use of elastic-plastic constitutive equations along with continuum damage mechanics became vital to better describe the mechanical behavior of concrete. The coupled damage to concrete plasticity by defining damage growth as a function of accumulated plastic strains [9] is considered.

The extension of microcracks plays a critical role in the inelasticity of concrete, as it results in the degradation of the elastic stiffness. In the models it is taken into account by introducing of damage variables. In the simples case a single scalar damage variable is used. It ranging from 0 for the undamaged material to 1 for completely damaged material. The constitutive equation of elastic-plastic material with scalar isotropic damage (introduced by Kachanov [12] and further developed by Rabotnov [13] and others) takes the following form:

$$
\boldsymbol{\sigma}=(1-D) \cdot{ }^{4} \mathbf{C}_{0}^{e} \cdot \cdot\left(\boldsymbol{\varepsilon}-\boldsymbol{\varepsilon}^{p}\right)={ }^{4} \mathbf{C}^{e} \cdot \cdot\left(\boldsymbol{\varepsilon}-\boldsymbol{\varepsilon}^{p}\right)
$$

where $\boldsymbol{\sigma}$ is Cauchy stress tensor, $D$ is the scalar stiffness degradation variable, $\boldsymbol{\varepsilon}$ is the strain tensor, $\boldsymbol{\varepsilon}^{p}$ is the plastic strain tensor, ${ }^{4} \mathbf{C}_{0}^{e}$ the initial (undamaged) elastic stiffness of the material, while ${ }^{4} \mathbf{C}^{e}=(1-D) \cdot{ }^{4} \mathbf{C}_{0}^{e}$ is the degraded elastic stiffness tensor. The effective stress tensor is defined by the relation $\overline{\boldsymbol{\sigma}}={ }^{4} \mathbf{C}_{0}^{e} \cdots\left(\boldsymbol{\varepsilon}-\boldsymbol{\varepsilon}^{p}\right)=\boldsymbol{\sigma} /(1-D)$.

The evolution of the scalar degradation variable is defined by function $D=D\left(\overline{\boldsymbol{\sigma}}, \widetilde{\varepsilon}^{p}\right)$, governed by a set of the effective stress tensor $\overline{\boldsymbol{\sigma}}$ and hardening (softening) variables $\widetilde{\varepsilon}^{p}$. In the used in further Lubliner model [9], the stiffness degradation is initially isotropic and defined by degradation variable $D_{c}$ in a compression zone and variable $D_{t}$ in a tension zone.

The cracking (under tension) and the crushing (under compression) in concrete are represented by increasing values of the hardening (softening) variables. These variables control the evolution of the yield surface and the degradation of the elastic stiffness. The 
yield function represents a surface in effective stress space $F\left(\overline{\boldsymbol{\sigma}}, \widetilde{\varepsilon}^{p}\right) \leq 0$ which determines the states of failure or damage.

Plastic flow is governed by a flow potential function $G(\overline{\boldsymbol{\sigma}})$ according to nonassociative flow rule:

$$
\dot{\boldsymbol{\varepsilon}}^{p}=\dot{\lambda} \frac{\partial G(\overline{\boldsymbol{\sigma}})}{\partial \overline{\boldsymbol{\sigma}}}
$$

The flow potential $G$ and yield function F are accepted in the forms:

$$
\begin{array}{r}
G=\sqrt{\left(f_{c}-m \cdot f_{t} \cdot \tan \beta\right)^{2}+3 \bar{J}_{2}}+\frac{1}{3} \bar{I}_{1} \cdot \tan \beta \\
F=\frac{1}{1-\alpha}\left(\sqrt{3 J_{2}}+\alpha \cdot I_{1}+\theta\left(\widetilde{\boldsymbol{\varepsilon}}^{p}\right)\left(\sigma_{\max }\right)-\gamma\left(-\sigma_{\max }\right)\right)-\sigma_{c}\left(\widetilde{\varepsilon}_{c}^{p}\right)
\end{array}
$$

where $f_{t}$ and $f_{c}$ are the uniaxial tensile and compressive strengths of concrete, respectively, $\beta$ is the dilation angle measured in the $\frac{1}{3} \bar{I}_{1}-\sqrt{3 \bar{J}_{2}}$ plane at high confining pressure, while $m$ is an eccentricity of the plastic potential surface, $\bar{I}_{1}=\mathbf{1} \cdot \overline{\boldsymbol{\sigma}}$ and $\bar{J}_{2}=\frac{1}{2} \operatorname{dev} \overline{\boldsymbol{\sigma}} \cdot \operatorname{dev} \overline{\boldsymbol{\sigma}}$.

Degradation of elastic modulus of concrete was shown in many experiments: tensile test [14-16], compressive test [17-21] of concrete.

\subsection{Interface constitutive model}

Cohesive behavior was used to describe destroying of reinforced-concrete bond. The available traction-separation model assumes initially linear elastic behavior followed by the initiation and evolution of the bond damage. The elastic behavior is written in terms of an elastic constitutive matrix that relates the nominal stresses to the nominal strains across the interface.

The nominal traction stress vector consists of three components: $t_{n}, t_{s}$ and $t_{t}$, which represent the normal and the two shear tractions respectively. The corresponding separations are denoted by $\delta_{n}, \delta_{s}$ and $\delta_{t}$.

The elastic behavior can then be written as

$$
\left\{\begin{array}{l}
t_{n} \\
t_{s} \\
t_{t}
\end{array}\right\}=\left[\begin{array}{ccc}
K_{n n} & K_{n s} & K_{n t} \\
K_{n s} & K_{s s} & K_{s t} \\
K_{n t} & K_{s t} & K_{t t}
\end{array}\right]\left\{\begin{array}{l}
\delta_{n} \\
\delta_{s} \\
\delta_{t}
\end{array}\right\} .
$$

The bond damage modeling allows to simulate the degradation and eventual failure of the bond between concrete body and steel rebar. The failure mechanism consists of two ingredients: a damage initiation criterion and a damage evolution law. 
The bond damage initiation refers to the beginning of degradation of the cohesive response at a contact point. The process of degradation begins when the contact stresses and/or contact separations satisfy certain damage initiation criteria.

Maximum stress criterion: the bond damage is assumed to initiate when the maximum contact stress ratio (as defined in the expression below) reaches a value of one. This criterion can be represented as

$$
\max \left\{\frac{\left\langle t_{n}\right\rangle}{t_{n}^{0}}, \frac{t_{s}}{t_{s}^{0}}, \frac{t_{t}}{t_{t}^{0}}\right\}=1
$$

Maximum separation criterion: the bond damage is assumed to initiate when the maximum separation ratio (as defined in the expression below) reaches a value of one:

$$
\max \left\{\frac{\left\langle\delta_{n}\right\rangle}{\delta_{n}^{0}}, \frac{\delta_{s}}{\delta_{s}^{0}}, \frac{\delta_{t}}{\delta_{t}^{0}}\right\}=1
$$

The bond damage evolution law describes the rate at which the cohesive stiffness is degraded once the corresponding initiation criterion is reached

A scalar bond damage variable, $\mathrm{D}_{\mathrm{b}}$, represents the overall damage at the contact point. It initially has a value of 0 . If damage evolution is modeled, $D_{b}$ monotonically increases from 0 to 1 upon further loading after the initiation of damage. The contact stress components are affected by the damage according to

$$
t_{n}=\left\{\begin{array}{ll}
\left(1-D_{b}\right) \bar{t}_{n}, & t_{n}>0 \\
\bar{t}_{n}, & t_{n} \leq 0
\end{array}, \quad t_{s}=\left(1-D_{b}\right) \bar{t}_{s}, \quad t_{t}=\left(1-D_{b}\right) \bar{t}_{t},\right.
$$

where $\bar{t}_{n}, \bar{t}_{\mathrm{s}}$ and $\bar{t}_{t}$ are the contact stress components predicted by the elastic tractionseparation behavior for the current separations without damage. To describe the evolution of bond damage under a combination of normal and shear separations across the interface, it is useful to introduce an effective separation [22] defined as

$$
\delta_{m}=\sqrt{\left\langle\delta_{n}\right\rangle^{2}+\delta_{s}^{2}+\delta_{t}^{2}} .
$$

An evolution of the bond damage variable $\mathrm{D}_{\mathrm{b}}$ was used for exponential softening, that reduces (in the case of damage evolution under a constant mode mix, temperature, and field variables) to

$$
D_{b}=1-\left\{\frac{\delta_{m}^{0}}{\delta_{m}^{\max }}\right\}\left\{1-\frac{1-\exp \left(-\alpha\left(\frac{\delta_{m}^{\max }-\delta_{m}^{0}}{\delta_{m}^{f}-\delta_{m}^{0}}\right)\right)}{1-\exp (-\alpha)}\right\} .
$$

In the expression above $\alpha$ is a non-dimensional parameter that defines the rate of damage evolution. 


\section{Results and Discussion}

The results of simulation of the pulling the reinforcing bar from the concrete block (see Fig. 1) are obtained using axisymmetric finite element (FE) model (FE mesh is shown in Fig. 2b). The numerical model consists of 4963 8-node quadrilateral elements (CAX8R). The computations were performed using the FE software package ABAQUS [11].

In order to determine the optimal strategy for solving the problem the six different models representing combinations of the bond behavior model and concrete model are considered and compared:

1. Ideal bond (bond model without discontinuity and cohesive behavior) and elastic model for concrete;

2. Bond model with cohesive behavior and elastic model for concrete;

3. Ideal bond and elastic-plastic model for concrete;

4. Bond model with cohesive behavior and elastic-plastic model for concrete;

5. Ideal bond and elastic-plastic-damage model for concrete;

6. Bond model with cohesive behavior and elastic-plastic-damage model for concrete.

Material parameters used in simulations are: concrete compression strength $R_{b}=18.5$ $\mathrm{MPa}$, tensile strength is $\mathrm{R}_{\mathrm{bt}}=1.55 \mathrm{MPa}$, Young modulus is $\mathrm{E}=30000 \mathrm{MPa}$, Poisson's ratio is $v=0.2$. To describe linear elastic traction-separation behavior the following parameters were used: $K_{n n}=1500 \mathrm{MPa} / \mathrm{mm}, K_{s s}=360 \mathrm{MPa} / \mathrm{mm}$, and $K_{t t}=360 \mathrm{MPa} / \mathrm{mm}$. Maximum stresses, which were used for damage initiation are: $t_{n}^{0}=110 \mathrm{MPa}, t_{s}^{0}=9 \mathrm{MPa}$, and $t_{t}^{0}=9 \mathrm{MPa}$. The elasticplastic model with isotropic hardening [23] with $\sigma_{02}=235 \mathrm{MPa}$ is used for steel rebar in FE computations.

Comparison of experimental data with simulation results of the pulling the reinforcing rebar from the concrete block for six considered models are shown in Fig. 4.

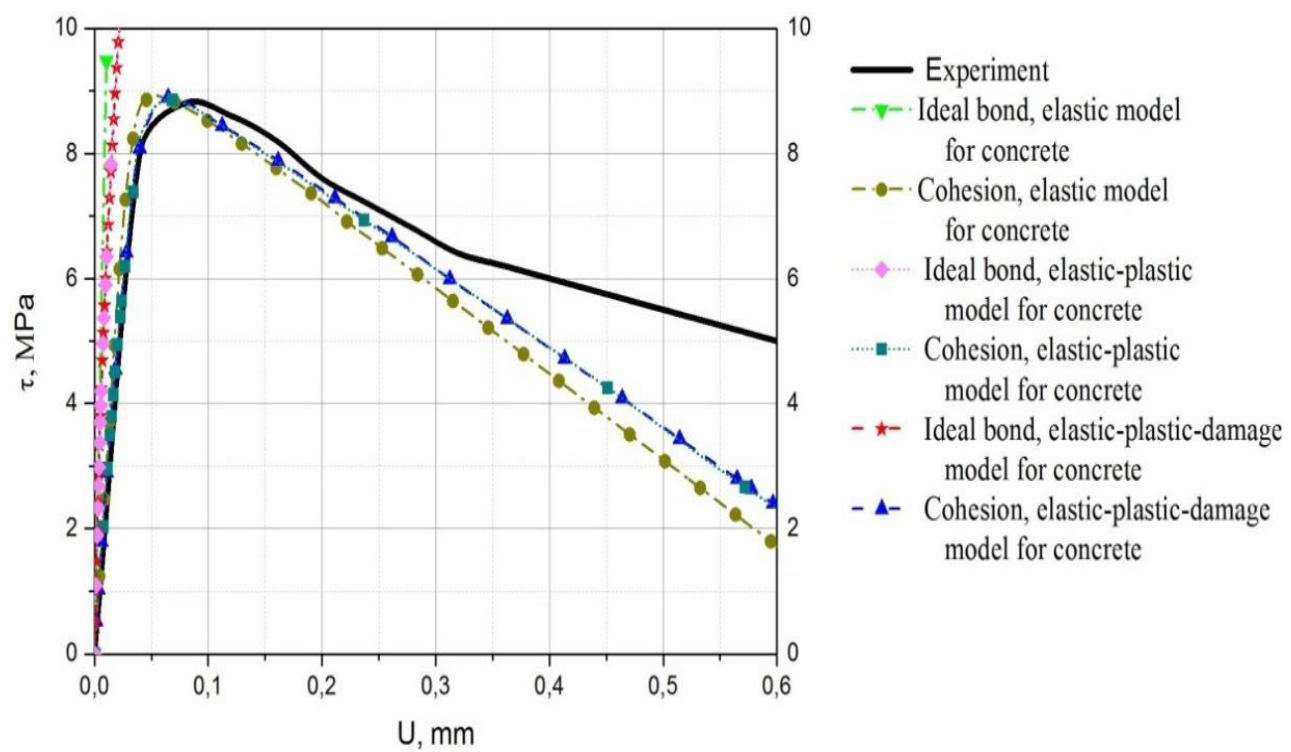

Fig. 4 Dependences of the shear stress in bond on the displacement of reinforcement.

All three models with ideal bond are unable to describe the appearance of a peak in dependence of the shear stress in bond on the displacement of reinforcement. Despite the concrete model, using the model of ideal bond without taking into account the effects of 
bond damage doesn't allow to describe correctly the process of pulling out of rebar from the concrete block. However using bond model [24] with cohesive behavior allows to describe the peak of shear stresses and rising branch on the diagram. Also it allows to simulate the degradation and eventual failure of the bond even with elastic model for concrete. As well as application of elastic model for concrete using the elastic-plastic model for concrete and the model of ideal bond doesn't allow to describe the pulling process. Using bond model with cohesive behavior gives near results for both elastic and elastoplastic models for concrete. There is no difference in results with using elastic-plastic and elastic-plastic-damage models for concrete. The obtained results point out on the dominant influence of accounting cohesion in bond model on pulling process description and weak influence of accounting model of concrete model. The proposed approach, which is based on direct FE modeling of reinforced concrete elements with account of nonlinear material model of concrete and cohesive behavior for interface between reinforcement and concrete, allows to describe correctly reinforced-concrete bond at pulling the rod out of the concrete body.

\section{References}

1. M. Ahmed Diab, E.Hafez Elyamany, A. Mostafa Hussein, M. Hazem Al Ashy, AEJ, 53(2), 355-371 (2014)

2. Y. Zhao, H. Lin, K. Wu, W. Jin, Constr. Build. Mater., 48, 348-359 (2013)

3. A. Michou, A. Hilaire, F. Benboudjema, G. Nahas, P. Wyniecki, Y. Berthaud, Engineering Structures, 101, 570-582 (2015)

4. A.V. Benin, A.S. Semenov, S.G. Semenov, Adv. Mat. Res., 831, 264-369 (2013)

5. A.V. Benin, A.S. Semenov, S.G. Semenov, Advances in Civil Engineering and Building Materials (CRC Press, Moscow, 2012)

6. RILEM/CEB/FIB. Recommendation on reinforcement steel for reinforced concrete. RC6. Bond test for reinforcement steel. 2. Pull-out tests (1983)

7. A.V. Benin, A.S. Semenov, S.G. Semenov, B.E. Melnikov, MCE, 5, 86-144 (2013)

8. A.B. Benin, A.S. Semenov, S.G. Semenov, B.E. Melnikov, MCE, 1(45), 23-40 (2014)

9. J. Lubliner, J. Oliver, S. Oller, E. Onate, Int. J. Solids Struct., 25(3), 299-326 (1989)

10. T. Jankowiak, T. Lodygowski, FCEE, 6, 53-69 (2005)

11. Abaqus/CAE User's Guide (2013)

12. L.M. Kachanov, Proc. Academy of Sci. SSSR., 8, 26-31 (1958)

13. Y.N. Rabotnov, Creep problems in structural members (NHPC, Amsterdam, 1969)

14. R.H. Evans, M.S. Marathe, Materials and Structures, 1(1), 61-64 (1968)

15. V.S. Gopalaratnam, P. Surendra Shah, ACI Journal proceedings, 82(3), 310-323 (1985)

16. H.W. Reinhardt, HERON, 29(2), 3-41 (1984)

17. I.D. Karsan, J.O. Jirsa, Proceeding of the American Society of Civil Engineers, 95(12), 2543-2563 (1969)

18. Mazars, Y. Berthaud, S. Ramtani, The unilateral Behaviour of Damaged Concrete, Eng . Fract. Mech., 35(4), 629-635 (1990)

19. S. Okamoto, S. Shiomi, K. Yamabe, Proceedings of Annual Architectural Instititute of Japan (AIJ) Convention, 1251-1252 (1976)

20. B.P. Sinha, K.H. Gerstle., L.G. Tulin, ACI J Proc., 61(2), 195-211 (1964)

21. Y.-H. Lee, K. Willam, ACI Materials Journal, 94/6, 457-471 (1997)

22. P.P. Camanho, C.G. Davila, NASA/TM, 211737, 1-37 (2002)

23. B.E. Melnikov, A.S. Semenov, S.G. Semenov, Proceedings of the A.N. Krylov Shipbuilding Research Institute, 53, 85-92 (2010)

24. T.S. Phan, P. Rossi, J.-L. Tailhan, Numerical modelling of the concrete/rebar bond, Cement and Concrete Composites, 59, 1-9 (2015) 